\title{
Performance Study of Multi-Path in VANETs and Their Impact on Routing Protocols
}

\author{
Jing Wu, Yuhao Wang ${ }^{\#}$ \\ Department of Electronic and Information Engineering, Nanchang University, Nanchang, China. \\ Email: "wangyuhao@ncu.edu.cn
}

Received March 16 ${ }^{\text {th }}, 2011$; revised April 7 th 2011; accepted April 18 ${ }^{\text {th }}, 2011$.

\begin{abstract}
Modeling complicated vehicular traffic behavior and analyzing effects on the communication performance of routing protocols taken by environmental factors have been a challenging task for the past several years. In this paper, we study the performance of multi-path routing protocol. Then, we investigate both multi-path and single-path routing protocol and analyze characteristics such as network connectivity, vehicle-node density etc. To better understand this phenomenon, we use mobility model with different parameters and evaluate the performance of routing protocols (Ad-hoc On-Demand Distance Vector and Ad-hoc On-demand Multipath Distance Vector routing protocols) through NS2 platform. The simulation results show the significance of multi-path on the throughput, loss and average delay of VANETs. The results of this paper may be used to study designing route protocols and applications of VANET.
\end{abstract}

Keywords: Vehicular Ad Hoc Networks (VANETs), Multi-Path, AODV, AOMDV

\section{Introduction}

Vehicular Ad hoc Networks (VANETs) as a new type of ad hoc network has been envisioned for inter-vehicle communications. With development of VANETs changing from military to civilian use, more and more applications are joined into VANETs. Routing losing makes the routing unreliable and instable, especially multimedia technology applied to VANETs. [1] has shown that in this type of network, the vehicles are the mobile nodes of the network. Vehicles can inform other vehicles of hazardous road conditions, traffic congestion, or sudden stops; furthermore, commercial services (e.g., data exchange, infotainment, rear-seat multiplayer games) can create an incentive for faster adoption of VANETs technology.

VANETs have mobility characteristics that distinguish them from the other MANETs. They have very high speed and limited degrees of freedom in vehicle nodes movement patterns. So VANETs have a highly dynamic topology due to the fast motions of vehicles. Further, the nodes do not have power constraints due to their access to the electrical system of the vehicle, which means energy of VANETs nodes is supplied by vehicles, and it is

\footnotetext{
${ }^{*}$ This work was supported in part by the Natural Science Foundation of Jiangxi Province for Youth under Grant 2010GQS0153 and 2009 GQS0070.
}

not limited resource [2]. The main aim of traditional routing protocols in VANETs is to discover a single path from source node to destination node. Because these routing protocols use a single path, they do not fully use a route discovery information. While in VANETs the mobility of nodes can easily lead to broken links, and a new round of route discovery will bring more delay and overhead [3]. Multi-path routing is defined between the source node and destination node creates multiple transmission paths, and allows nodes to choose how to use these paths $[4,5]$. Source mode to the destination node has multi-paths, making the routing protocol to better adapt to VANETs dynamics, topology and unpredictable characteristics [6].

Since all the vehicle nodes have routing function in VANETs, there exist multi-path from the source to the destination, so it is more suitable to adopt multi-path routing protocol. On the other hand, using multi-path routing can efficiently enhance network bandwidth, fairly utilize network resources and prolong network lifetime. So in this paper we introduce multi-path routing protocol in VANETs, which could build multi-path between source and destination nodes during route discovery. We compare performance of single path and multi-path protocols under one special mobility model with different value of environmental factors. This paper chooses a 
widely-used network simulator NS2, fixing bugs and incorporating assumptions for fair comparisons into the implementations of different routing protocols and the simulator itself.

The paper is structured as follows. Section II we discuss the system model. Section III shows simulation scenarios and parameters. Section VI evaluates the performance of routing protocols. The paper concludes in Section V.

\section{System Model}

In this section, we review some research works on multipath analyze multi-path routing protocol and adopt routing protocol AOMDV [7] in VANETs. Then we will introduce the works on the following three parts.

\subsection{Multi-Path Type Selection}

Multi-path can be divided into three categories based on the relevance of the node: node disjoint multi-path, linkdisjoint multi-path and non-disjoint multi-path.

\subsubsection{Node Disjoint Multi-Path}

Node disjoint multi-path routing is also known as completely unrelated routing, the path that is not shared between the nodes or links. Shown in Figure 1, the roads between the source node $\mathrm{S}$ and destination node D (SN1-N2-D, S-N3-N4-D) are node disjoint routes.

\subsubsection{Link Disjoint Multi-Path}

Link disjoint multi-path refers to the path is not shared between the link, but may have common nodes. Shown in Figure 2, the path between source node $S$ and destination node D (S-N1-N3-N4-D, S-N2-N3-N5-D) are the link disjoint routes, and the node N3 is the common node.

\subsubsection{Non-Disjoint Multi-Path}

Non-disjoint multi-path refers to path that has shared nodes and common links. Shown in Figure 3, S-N1-N3N4-N5-D, S-N2-N3-N4-N6-D are non-disjoint routes, N3-N4 is the common link.

Non-disjoint multi-path takes up less resources compared with disjoint multi-path, because it has both shared link and nodes. At the some network density, route search is much easier for non-disjoint, cause binding of route search for disjoint multi-path is much stronger. But it is precisely because non-disjoint multi-path has shared link and nodes, makes its fault tolerance worse. In the three types of routing, fault tolerance of node disjoint multipath is the strongest, then link disjoint multi-path, and non-disjoint is the worst. If the shared node interrupted due to mobile and other reasons, in the link disjoint multi-path, the paths contain the shared node will all failed, while in node disjoint multi-path, it will not produce a

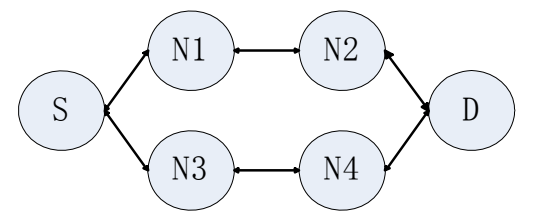

Figure 1. Node disjoint multi-path.

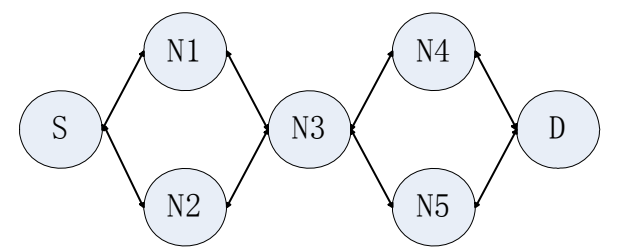

Figure 2. Link disjoint multi-path.

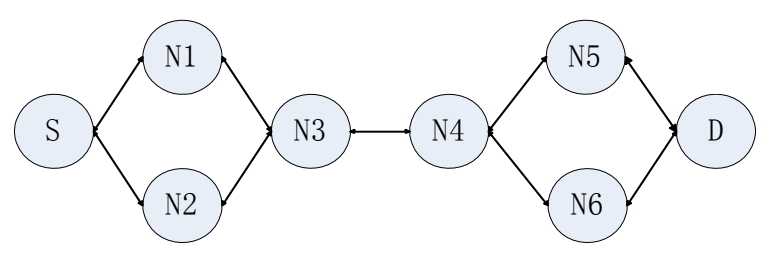

Figure 3. Non-disjoint multi-path.

chain reaction due to the independence of the link. Usually in the network with relatively large density, use the node disjoint multi-path routing. We use link disjoint multi-path routing in the network with relatively sparse density, and do not use non-disjoint.

\subsection{Multi-Path Routing Protocols Analysis}

Multi-path routing is defined to provide multi-path for any pair of nodes, and allows the host node or application to choose how to use these paths. Multi-path routing algorithm provides multi-path, and ensure the data path through the path to the destination. The literature [8,9] theoretically proved that the on-demand multi-path has a longer survival time, more reliable path routing information, a good performance, and reduce some congestion. So in recent years, multi-path has gained much attention.

Currently, the study of multi-path routing protocol mainly focuses on two aspects, DSR and AODV routing protocol improvements. Then the study based on DSR and AODV multi-path routing protocol provides a basis for improving traditional single path routing protocol in VANETs $[10,11]$.

AOMDV is based on the AODV routing protocol and extends the AODV, each route discovery can make multi-path between source and destination nodes, and ensure that these paths do not cross and no ring. Compared with other on-demand multi-path routing protocol, AOMDV has three new features: First, the cost of node collaboration is low; Second, do not use source routing strategy, ensure multiple alternative paths do not cross by 
distributed computing; Third, AOMDV use as much as possible already existing alternative path information, calculate alternative paths based on AODV minimum overhead. Compared with AODV, AOMDV did not use any special routing control packets, the only additional cost is RREQ packet, RERP packet and the additional fields used in RRER packet.

\subsection{The Integration of Multi-Path and VANETs}

Multi-path routing is supposed to reduce the end-to-end packet delay and increase the packet delivery ratio. Therefore, it can also improve the packet delivery ratio in VANETs when the mobility of relaying vehicles is unknown. In VANETs multi-path routing need to find out the node-disjoint path between the given source and destination nodes $\mathrm{S}$ and D. For single path, we choose shortest path between the source and the destination. As the distance increases, the end-to-end packet delivery ratio quickly drops, and the delay increases. Therefore, the shortest path achieves the best performance in terms of delay. The S-D distance is defined as the hop count of the shortest path between the source and destination nodes. The intriguing question is whether the node-disjoint multi-path routing really helps. In this paper, we examine the performance of node-disjoint multi-path routing in VANETs.

\section{Simulation Scenarios and Parameters}

Communication between vehicles is frequently mentioned as a target for ad hoc routing protocols, there have previously been not many studies on how the specific movement patterns of vehicles may influence the protocol performance and applicability [12-14]. Typically the behavior of routing protocols for VANET is analyzed based on the assumption that the nodes in the network follow the random waypoint mobility model. In this model each node randomly selects a waypoint in the simulation and moves from its current location to the waypoint with a random but constant speed.

In this paper, we assume vehicular environments of urban area, in order to have an accurate evaluation of such condition, the mobility model is adapted to the road linear characteristics. Thus the performance of routing protocol is simulated reasonably and the conclusion has some value in the simulation and practical application of VANET. The parameters of simulation are shown in Table 1.

This paper considers the opposite moving direction of vehicles on the road, and there are 10 vehicles on one road in each direction. The inter-vehicle distance (IVD) is $27 \mathrm{~m}$ which corresponds to the expected security distance related to the legal limitation speeds of $50 \mathrm{~km} / \mathrm{h}$. This paper uses shadow propagation model which is the basic and simple model of transmission that provides a simplified computation environment. The size of the packets is equal to 1000B. The transport protocol is UDP, due to the mobility of vehicles, a large area should be defined. The scene is shown in Figure 4.

\section{Simulation Results Analysis}

In this section, we present some numerical results regarding the analysis in this paper, together with simulation results, to confirm the accuracy of the analysis.

First, we present the simulation results for AODV and AOMDV routing protocols under 40 vehicle-nodes situation. As we can see in Figure 5 that two kinds of routing protocols have similar characteristics, with the vehicle-node speed increased the network throughput decreased. But we can find that vehicle-node speed has little effect on network throughput when use AOMDV

Table 1. The parameters of simulation.

\begin{tabular}{lc}
\hline Simulation area $\left(\mathrm{m}^{2}\right)$ & $1000 * 1000$ \\
Simulation time (s) & 800 \\
Number of nodes & 40,80 \\
Inter-vehicle distance(m) & 27 \\
Data Type & CBR \\
Packet size (bytes) & 1000 \\
MAC protocol & IEEE802.11 \\
Link rate (Mbps) & 54 \\
Propagation model & Shadow \\
Path loss exponent & 3.8 \\
Shadowing deviation $(\mathrm{dB})$ & 2.0 \\
Channel frequency $(\mathrm{Hz})$ & $2.4 \mathrm{e} 9$ \\
\hline
\end{tabular}

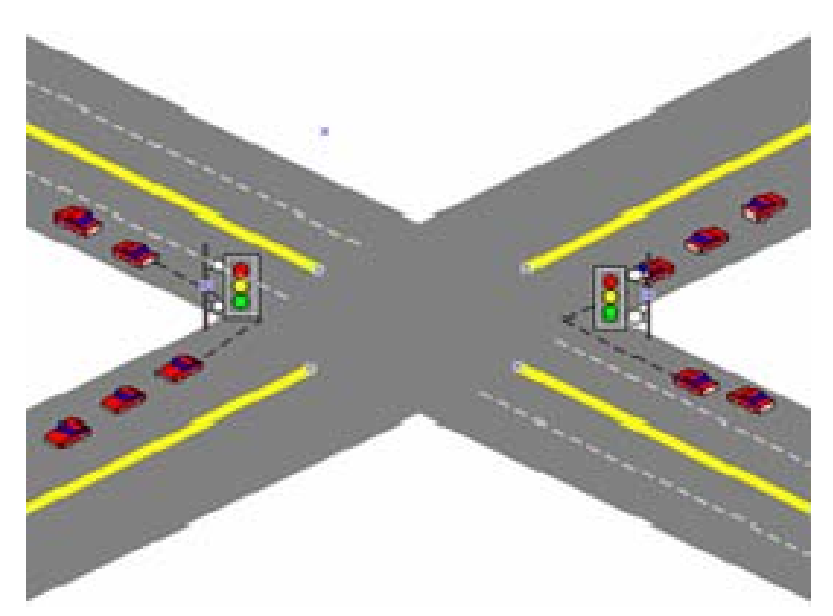

Figure 4. Mobile scenarios. 


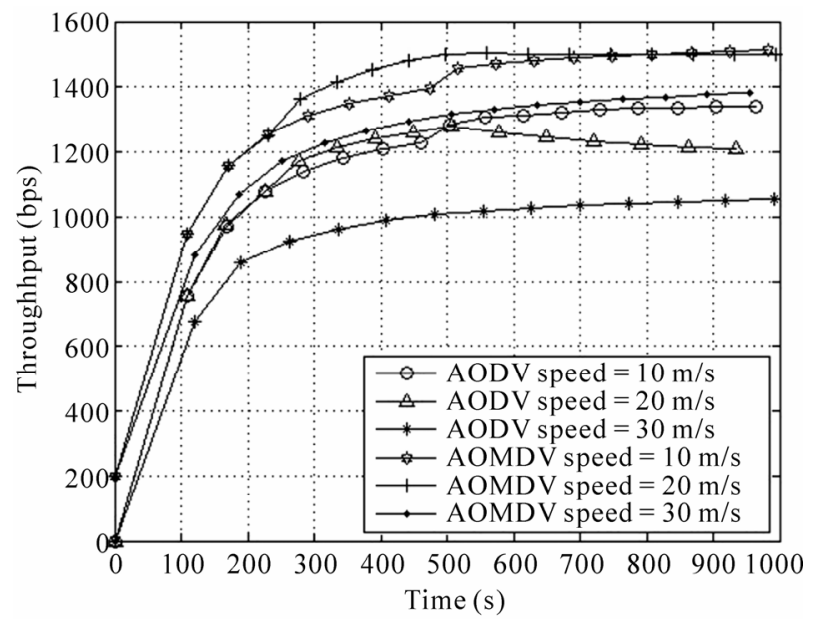

Figure 5. Simulation with 40 vehicle nodes.

routing protocol. Besides we compare vehicle-node speed $=10 \mathrm{~m} / \mathrm{s}, 20 \mathrm{~m} / \mathrm{s}, 30 \mathrm{~m} / \mathrm{s}$ these three situations, we find that the performance gets worse with the vehicle-node speed increased. This is due to the possibility of failed route path increases with vehicle-node speed increased. So adopting AOMDV routing protocol in VANETs could fit different values of vehicle-node speed better, and the performance of vehicle network throughput is more stable.

Next, we present the results for AODV and AOMDV routing protocols under 80 vehicle-nodes situation in Figure 6. Also we can see that the performance of vehicle network throughput gets worse as the vehicle-node speed increased. Compared with Figure 5, the throughput of network is obviously reduced. With the vehiclenode density increased, the performance of network has a corresponding decline. But AOMDV performs better than AODV in both throughput and stability.

Then we simulate packet loss rate and average delay in both AODV and AOMDV routing protocol under different vehicle-node density. From Tables 2-5, we can see that in vehicular Ad hoc Network packet loss rate and average end to end delay can substantially reduce when using AOMDV routing protocol.

\section{Conclusions}

In this paper we have evaluated the performance of AOMDV routing protocol in vehicular Ad hoc Network using NS-2. AODV and AOMDV separately represent single path and multi-path routing protocols. We bring multi-path routing protocol in VANETs, and analyze the performance of single and multi-path routing protocols by measuring throughput, packet loss rate and average end to end delay. The results obtained show that the multi-path (node disjoint) has the ability to provide satisfactory packet loss rate and average end to end delay.

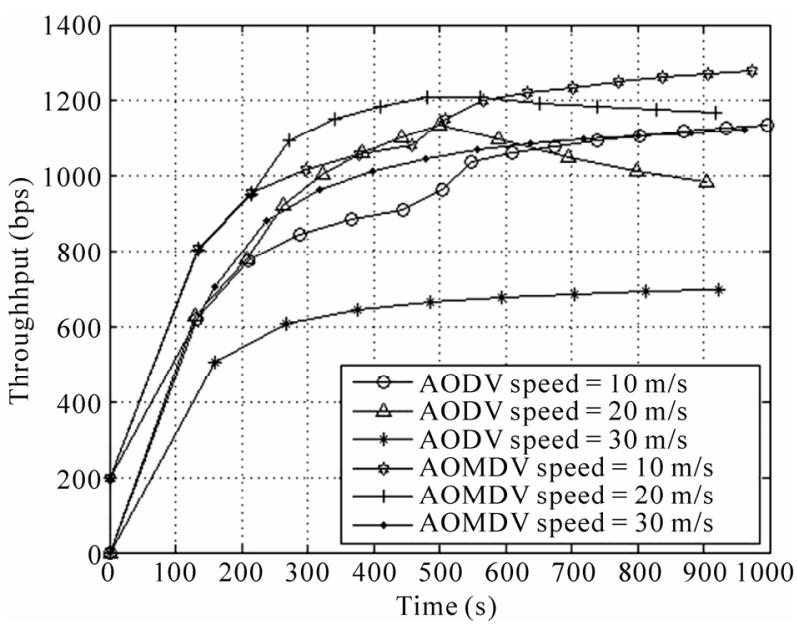

Figure 6. Simulation with 80 vehicle nodes.

Table 2. Loss with $\mathbf{4 0}$ vehicle nodes.

\begin{tabular}{cccc}
\hline Speed (m/s) & 10 & 20 & 30 \\
\hline AODV & $40.53 \%$ & $40.66 \%$ & $42.67 \%$ \\
AOMDV & $20.73 \%$ & $21.43 \%$ & $21.43 \%$ \\
\hline
\end{tabular}

Table 3. Loss with 80 vehicle nodes.

\begin{tabular}{cccc}
\hline Speed (m/s) & 10 & 20 & 30 \\
\hline AODV & $18.64 \%$ & $18.04 \%$ & $21.36 \%$ \\
AOMDV & $6.39 \%$ & $6.85 \%$ & $8.78 \%$ \\
\hline
\end{tabular}

Table 4. Average end to end delay with 40 vehicle nodes.

\begin{tabular}{cccc}
\hline Speed (m/s) & 10 & 20 & 30 \\
\hline AODV (s) & 0.080507 & 0.102453 & 0.038062 \\
AOMDV (s) & 0.011946 & 0.027289 & 0.006398 \\
\hline
\end{tabular}

Table 5. Average end to end delay with 80 vehicle nodes.

\begin{tabular}{cccc}
\hline Speed $(\mathrm{m} / \mathrm{s})$ & 10 & 20 & 30 \\
\hline AODV (s) & 0.248203 & 0.162907 & 0.032375 \\
AOMDV (s) & 0.028180 & 0.010789 & 0.025905 \\
\hline
\end{tabular}

This paper just simulated the scene of crossroad, considered vehicle node speed and density. The results of this paper may be used to study the performance of different routing algorithms, throughput, or packet loss rate in VANETs.

\section{REFERENCES}

[1] M. Boban, O. K. Tonguz and J. Barros, "Unicast Communication in Vehicular Ad Hoc Networks: A Reality 
Check,” IEEE Communications Letters, Vol. 13, No. 12, 2009, pp. 995-997. doi:10.1109/LCOMM.2009.12.091497

[2] M. Khabazian and M. K. M. Ali, "A Performance Modeling of Connectivity in Vehicular Ad Hoc Networks," IEEE Transactions on Vehicle Technology, Vol. 57, No. 4, 2008, pp. 2440-2450. doi:10.1109/TVT.2007.912161

[3] C. H. Foh, G. Liu, B. S. Lee, B. Seet, et al., "Network Connectivity of One-Dimensional MANETs with Random Waypoint Movement," IEEE Communications Letters, Vol. 9, No. 1, 2005, pp. 31-33.

[4] X. X. Huang and Y. G. Fang, "Performance Study of Node-Disjoint Multipath Routing in Vehicular Ad Hoc Networks,” IEEE Transactions, Vol. 58, No. 4, 2009, pp. 1942-1950.

[5] R. X. He, H. Rutagemwa and X. M. Shen "Differentiated Reliable Routing in Hybird Vehicular AD-Hoc Networks," Proceedings of IEEE International Conference on Communications, Beijing, 2008, pp. 2353-2358.

[6] P. Pham, S. Perreau. "Performance Analysis of Reactive Shortest Path and Multipath Routing Mechanism with Load Balance," Proceedings of the 22nd Annual Joint Conference of the IEEE Computer and Communications, San Francisco, Vol. 1, 30 March-3 April 2003, pp. 251259.

[7] S. R. Das. "On Demand Multipath Distance Vector Routing For Ad Hoc Network," Proceedings of IEEE International Conference on Network Protocol (ICNP), Mission Inn, 11-14 November 2001, pp. 14-23.

[8] NASIPURIA, DAS SR. “On-Demand Multipath Routing for Mobile Ad Hoc Networks," Proceedings of Computer Communications and Networks Conference, IEEE Press, New Orelans, 1999, pp. 64-70.

[9] P. Johanson, T. Larsson, N. Hedman, et al., "Scenario Based Performance Analysis of Routing Protocols for Mobile Ad Hoc Networks," Proceeding of the ACM/IEEE International Conference on Mobile Computing and Networking, IEEE Press, SanAn-tonio, 1999, pp. 195- 206.

[10] C.-S. Wu, S.-C. Hu and C.-S. Hsu, "Design of Fast Restoration Multipath Routing in VENETs," Proceedings of IEEE International Computer Symposium, Tainan, 16-18 December 2010, pp. 73-78.

[11] J. Fukuyama, "A Probabillistic Protocol for Multi-Hop Routing in VANETs," Proceedings of IEEE International Conference on Communications Workshops, Dresden, 14-18 June 2009, pp. 1-6. doi:10.1109/ICCW.2009.5208064

[12] W.-H. Liao, S.-L. Wang and J.-P. Sheu, "A Multi-Path QoS Routing Protocol in a Wireless Mobile Ad Hoc Network,” Telecommunication Systems, Vol. 19, No. 3-4, 2002, pp. 329-347. doi:10.1023/A:1013838304991

[13] S.-J. Lee and M. Gerla, "Split Multipath Routing with Maximally Disjoint Paths in Ad Hoc Networks,” Proceedings of IEEE International Conference on Communications, 11-14 June 2001, pp. 3201-3205.

[14] R. Zoican and D. Galatchi, "Analysis and Simulation of a Predictable Routing Protocol for VANETs,” Proceedings of the 9th International Symposium on Electronics and Telecommunications, Timisoara, 11-12 November 2010, pp. 153-156. 